\title{
Cancer Bioinformatics Grid
}

National Cancer Institute

\section{Source}

National Cancer Institute. Cancer Bioinformatics Grid. NCI Thesaurus. Code C49046.

A voluntary network or grid connecting individuals and institutions to enable the sharing of data and tools, creating a World Wide Web of cancer research. The goal is to speed the delivery of innovative approaches for the prevention and treatment of cancer. The infrastructure and tools created by caBIG also have broad utility outside the cancer community. caBIG is being developed under the leadership of the National Cancer Institute's Center for Bioinformatics. 